\title{
Association study of 21 circadian genes with bipolar I disorder, schizoaffective disorder, and schizophrenia
}

\author{
Hader A Mansoura, Michael E Talkowski ${ }^{\mathrm{a}}, \mathrm{b}$, Joel Wood ${ }^{\mathrm{a}}$, Kodavali V Chowdaria ${ }^{\mathrm{a}}$, Lora \\ McClain $^{\mathrm{a}}$, Konasale Prasad ${ }^{\mathrm{a}}$, Debra Montrose ${ }^{\mathrm{a}}$, Andrea Fagiolini ${ }^{\mathrm{C}}$, Edward S Friedman ${ }^{\mathrm{a}}$, \\ Michael H Allen ${ }^{d}$, Charles L Bowden ${ }^{e}$, Joseph Calabrese ${ }^{f}$, Rif S El-Mallakh ${ }^{g}$, Michael \\ Escamilla $^{e}$, Stephen V Faraone ${ }^{h}$, Mark D Fossey ${ }^{i}$, Laszlo Gyulaij, Jennifer M Loftis ${ }^{k, l}$, Peter \\ Hauser ${ }^{k, l, m}$, Terence A Ketter ${ }^{n}$, Lauren B Marangell ${ }^{\circ}$, David J Miklowitz ${ }^{\mathrm{p}}$, Andrew A \\ Nierenberg ${ }^{q}$, Jayendra Patel ${ }^{r}$, Gary S Sachs ${ }^{s}$, Pamela Sklar ${ }^{t}$, Jordan W Smoller ${ }^{\mathrm{u}}$, Nan \\ Lairdv, Matcheri Keshavan $^{\mathrm{a}}$, Michael E Thasew ${ }^{\mathrm{w}}$, David Axelson ${ }^{\mathrm{a}}$, Boris Birmaher ${ }^{\mathrm{a}}$, David \\ Lewis $^{\mathrm{a}}$, Tim Monk ${ }^{\mathrm{a}}$, Ellen Frank ${ }^{\mathrm{a}}$, David J Kupfera ${ }^{\mathrm{a}}$, Bernie Devlin ${ }^{\mathrm{a}}$, and Vishwajit L \\ Nimgaonkara,b
}

\begin{abstract}
(C) 2009 The Authors.
Corresponding author: Vishwajit L. Nimgaonkar, M.D., Ph.D., Department of Psychiatry and Human Genetics, University of Pittsburgh School of Medicine, Graduate School of Public Health, Western Psychiatric Institute and Clinic, 3811 O'Hara Street, Room 441, Pittsburgh, PA 15213, USA, Fax: (412) 246-6350, nimga+@ pitt.edu.

Disclosures

$\mathrm{AF}$ is/has been a speaker and/or a consultant for Bristol-Myers Squibb, Eli Lilly \& Co., Pfizer, Boeringher Ingelheim Jannssen, Novartis, and Takeda. ESF has received grant/research funding from NIMH, Sanofiaventis, Cyberonics, Novartis, and Northstar; and has served as a consultant for Pfizer. JC has received federal funding from the Department of Defense, Health Resources Services Administration and NIMH; has received grant/research support from Abbott, AstraZeneca, Bristol-Myers Squibb, Cephalon, the Cleveland Foundation, Eli Lilly \& Co., GlaxoSmithKline, Janssen, NARSAD, Repligen, Stanley Medical Research Institute, Takeda, and Wyeth; has been an advisory board member for Abbott, AstraZeneca, Bristol-Myers Squibb, Dainippon Sumitomo, EPI-Q, Inc., Forest, France Foundation, GlaxoSmithKline, Janssen, Johnson \& Johnson, Lundbeck, Neurosearch, OrthoMcNeil, Otsuka, Pfizer, Repligen, Schering-Plough, Servier, Solvay, Supernus Pharmaceuticals, Synosia, and Wyeth; and has been involved in CME activities supported by AstraZeneca, Bristol-Myers Squibb, the France Foundation, GlaxoSmithKline, Janssen, Johnson \& Johnson, Sanofiaventis, Schering-Plough, Pfizer, Solvay, and Wyeth. RSE-M is on the speakers bureau for Abbott, AstraZeneca, Bristol-Myers Squibb, Eli Lilly \& Co., and Pfizer; he reports no conflicts as far as this paper is concerned. PH has been on the speakers bureau for AstraZeneca and Jazz Pharmaceuticals; and has received an investigator-initiated foundation grant from Janssen Pharmaceuticals. TAK has received grant/research support from Abbott, AstraZeneca, Bristol-Myers Squibb, Cephalon, Eli Lilly \& Co,

GlaxoSmithKline, Pfizer, Repligen, and Wyeth; has served as a consultant for Abbott, AstraZeneca, Bristol-Myers Squibb, Dainippon Sumitomo Pharma, Eli Lilly \& Co., GlaxoSmithKline, Janssen, Jazz Pharmaceuticals, Novartis, Organon International, Inc., a part of Schering-Plough Corp., Solvay, Valeant Pharmaceuticals, Vanda Pharmaceuticals, Wyeth, and XenoPort, Inc.; has received lecture honoraria from Abbott, AstraZeneca, Bristol-Myers Squibb, Eli Lilly \& Co., GlaxoSmithKline, Noven Pharmaceuticals, Otsuka Pharmaceuticals, and Pfizer; and his spouse is employed by Johnson \& Johnson. LBM is an employee of and owns stock in Eli Lilly \& Co. The work presented here represents her views and not those of Eli Lilly \& Co. MET has received grant support from Eli Lilly \& Co., GlaxoSmithKline, NIMH, and Sepracor, Inc.; has served as an advisor/consultant for AstraZeneca, Bristol-Myers Squibb, Eli Lilly \& Co., Forest Laboratories, GlaxoSmithKline, Janssen, MedAvante, Inc., Neuronetics, Inc., Novartis, Schering-Plough, Shire US, Inc., Supernus Pharmaceuticals, Transcept Pharmaceuticals, and Wyeth; has served on the speakers bureau for AstraZeneca, Bristol-Myers Squibb, Eli Lilly \& Co., and Wyeth; holds equity in MedAvante, Inc., has received royalties from American Psychiatric Publishing, Inc., Guilford Publications, Herald House, and W.W. Norton \& Company; and his spouse is employed at Advogent (Formerly Cardinal Health) as of 4/17/09. BB has during the past 12 months been invited to participate at forums sponsored by Forest Laboratories, Inc., and Shire Pharmaceuticals; and has received or will receive royalties from Random House, Inc., and Lippincott Williams \& Wilkins. DL currently receives investigator-initiated research support from the BMS Foundation, Bristol-Myers Squibb, Curridium Ltd., and Pfizer; and in 2007-2009 has served as a consultant for AstraZeneca, Bristol-Myers Squibb, Hoffman-Roche, Eli Lilly \& Co., Merck, and Neurogen. EF serves on the Servier International Advisory Board and has received royalties from Guilford Publications. HAM, MET, JW, KVC, LM, KP, DM, MHA, CLB, ME, SVF, MDF, LG, JML, DJM, AAN, JP, GSS, PS, JWS, NL, MK, DA, TM, DJK, BD, and VLN do not have any conflict of interest to report in connection with this manuscript.
\end{abstract}

Supporting information

Additional supporting information may be found in the online version of this article

Please note: Wiley-Blackwell is not responsible for the content or functionality of any supporting materials supplied by the authors. Any queries (other than missing material) should be directed to the corresponding author for the article. 
aDepartment of Psychiatry, University of Pittsburgh School of Medicine, Western Psychiatric Institute and Clinic

${ }^{b}$ Department of Human Genetics, Graduate School of Public Health, University of Pittsburgh, Pittsburgh, PA, USA

'Department of Neuroscience, University of Siena School of Medicine, Siena, Italy

dDepartment of Psychiatry, University of Colorado Depression Center, Denver, CO

eDepartment of Psychiatry, University of Texas Health Science Center at San Antonio, San

Antonio, TX

fDepartment of Psychiatry, Case University School of Medicine, Mood Disorders Program, University Hospitals of Cleveland, Cleveland, $\mathrm{OH}$

gDepartment of Psychiatry and Behavioral Sciences, University of Louisville School of Medicine, Louisville, KY

hDepartment of Psychiatry and Human Behavior, SUNY Upstate Medical University, Syracuse, NY

'Department of Psychiatry, University of Oklahoma-Tulsa, Tulsa, OK

iDepartment of Psychiatry, University of Pennsylvania Medical Center, Philadelphia, PA

kBehavioral Health \& Clinical Neurosciences Division, Portland VA Medical Center, Oregon

Health and Science University, Portland, OR

'Department of Psychiatry, Oregon Health and Science University, Portland, OR

mDepartment of Behavioral Neuroscience, Oregon Health and Science University, Portland, OR

nBipolar Disorders Clinic, Department of Psychiatry and Behavioral Sciences, Stanford University School of Medicine, Stanford, CA

${ }^{\circ}$ Eli Lilly and Company, US Medical Division, Indianapolis, IN

PDepartment of Psychology, University of Colorado, Boulder, CO

'Clinical Depression and Research Program, Department of Psychiatry Harvard Medical School, Massachusetts General Hospital, Boston

rSchizophrenia Research Program, Bipolar Disorder Program and Center for Psychopharmacology Research and Treatment, Department of Psychiatry, University of Massachusetts Medical School, Worcester

sBipolar Clinic and Research Program, Department of Psychiatry, Harvard Medical School, Massachusetts General Hospital, Boston, MA

tPsychiatric and Neurodevelopmental Genetics Unit, Center for Human Genetic Research, Department of Psychiatry, Massachusetts General Hospital and Harvard Medical School, Boston, MA

uDepartment of Psychiatry, Psychiatric Genetics Program in Mood and Anxiety Disorders, Massachusetts General Hospital, Boston, MA

vDepartment of Biostatistics, Harvard School of Public Health, Boston, MA

wUniversity of Pennsylvania School of Medicine, Philadelphia VA Medical Center, Philadelphia, PA, USA

\section{Abstract}


Objective-Published studies suggest associations between circadian gene polymorphisms and bipolar I disorder (BPI), as well as schizoaffective disorder (SZA) and schizophrenia (SZ). The results are plausible, based on prior studies of circadian abnormalities. As replications have not been attempted uniformly, we evaluated representative, common polymorphisms in all three disorders.

Methods-We assayed 276 publicly available 'tag' single nucleotide polymorphisms (SNPs) at 21 circadian genes among 523 patients with BPI, 527 patients with SZ/SZA, and 477 screened adult controls. Detected associations were evaluated in relation to two published genome-wide association studies (GWAS).

Results-Using gene-based tests, suggestive associations were noted between EGR3 and BPI (p $=0.017)$, and between $N P A S 2$ and SZ/SZA $(\mathrm{p}=0.034)$. Three SNPs were associated with both sets of disorders (NPAS2: rs13025524 and rs11123857; RORB: rs10491929; $\mathrm{p}<0.05$ ). None of the associations remained significant following corrections for multiple comparisons.

Approximately $15 \%$ of the analyzed SNPs overlapped with an independent study that conducted GWAS for BPI; suggestive overlap between the GWAS analyses and ours was noted at ARNTL.

Conclusions-Several suggestive, novel associations were detected with circadian genes and BPI and SZ/SZA, but the present analyses do not support associations with common polymorphisms that confer risk with odds ratios greater than 1.5. Additional analyses using adequately powered samples are warranted to further evaluate these results.

\section{Keywords}

association; bipolar disorder; circadian; gene; schizoaffective disorder; schizophrenia

Family, twin, and adoption studies suggest heritability estimates of 60-80\% for bipolar I disorder (BPI), schizophrenia (SZ), and schizoaffective disorder (SZA) (1-3). The mode of inheritance of BPI has been difficult to define, but current analyses suggest multiple interacting loci contributing to BPI liability (4). This complexity may explain the inconsistencies that led to prior gene-mapping efforts. The traditional approach, called linkage analysis, involves the analysis of shared genomic regions among members of multiply affected families. Identification of such shared (linked) regions can help localize diseased genes. Though several genome-wide scans have now been completed, they differ not only with respect to ascertainment criteria, but also marker density, sample sizes, and method of analysis. Nevertheless, a number of groups have reported linkage signals in overlapping regions. Recent meta-analyses have identified several plausible regions for BPI, including chromosomes 13q, 22q, 9p22.3-21.1, 10q11.21-22.1, and 14q24.1-32.12 (5, 6). These linked regions have not been identified in all studies. The failure to replicate these results may reflect insufficient power, differing designs, the presence of genetic heterogeneity, or the absence of a susceptibility locus in the region.

Another complementary approach for gene mapping, called association analysis, might arguably hold greater promise (7). Association studies involve comparison of genetic polymorphisms among cases and suitable controls. Case-control differences in the frequency of alleles (variants) of particular polymorphisms may help pinpoint etiological factors. Historically, case-control association studies have focused on relatively small genomic regions in contrast to linkage studies. To improve the chances of detecting meaningful associations, researchers have selected particular genes whose products have been implicated in other avenues of bipolar research. This approach is called the "candidate gene' strategy. Among dozens of candidate genes that have been investigated, consistent associations have been difficult to detect (8). These inconsistent results may be related to variation in ascertainment, phenotype definition, and control selection, limited power 
(studies have typically included fewer than 200 cases or families), and possible confounding by population substructure. Another reason could be that the initial report was simply a false positive.

Among different types of polymorphisms used for gene-mapping studies, bi-allelic single nucleotide polymorphisms (SNPs) have come to the fore. The SNPs have been utilized to evaluate associations across the genome simultaneously. Such genome-wide association studies (GWAS) have helped identify replicable associations for several common multifactorial diseases, including type I and type II diabetes mellitus, Crohn's disease, agerelated macular degeneration, and several types of cancers (9). In view of the emerging success in other diseases, GWAS in SZ and BPI are in progress (9-15).

Several lines of evidence point to circadian dysfunction in BPI pathogenesis $(16,17)$. Patients with BPI display disrupted circadian function, including changes in sleep, appetite, and hormonal secretions, especially during relapse $(18,19)$. Disruption in circadian and social rhythms may precede the onset of manic episodes $(20,21)$. Clinical stabilization of bipolar symptoms is usually associated with normalization of the disrupted circadian function. Indeed, mood stabilizers may exert therapeutic effects by modulating circadian function (22-26).

A pathogenic role for circadian dysfunction in BPI can be investigated using genetic association studies. Several circadian gene polymorphisms have been investigated, with mixed results (27-31). Haplotype-based analyses suggest associations at Bmal I (ARNTL) and Period 3 (PER3) (32), as well as PER3 (31), with nonsignificant associations at PER2 and $C r y 1(32,33)$. Another recent study that analyzed 10 circadian genes reported interactions between SNPs at BHLHB2, CSNK1E, and CLOCK (34). The glycogen synthase kinase $3-\beta$ gene (GSK3- $\beta$ ) has also been extensively investigated in relation to BPI because it regulates the molecular clock in the suprachiasmatic nucleus (SCN) $(35,36)$. A number of association studies of GSK3 and bipolar disorder have been published with inconsistent results. While some studies have found associations $(27,28)$, others did not (29, $30,37)$. Circadian-rhythm abnormalities in schizophrenia were also reported. Martin et al. (38) showed that elderly schizophrenia patients had disrupted sleep and circadian rhythms compared with age- and gender-matched normal comparison subjects, while Wirz-Justice et al. (39) attributed circadian dysfunction to medication effects. Genetic associations between circadian genes and SZ/SZA have been examined less frequently. We have reported suggestive associations at ARNTL, PER3, and Timeless (TIMELESS), based on initial analyses of SNPs from eight circadian genes among patients with BPI and SZ/SZA and among controls, comprising over 1,450 individuals (40).

Since most published studies investigated disparate groups of SNPs in selected circadian genes, it is uncertain whether the reported results are robust. Here, we report on a systematic genetic association study. An international effort called the HapMap project has set out to identify and catalogue common SNPs across the human genome (http://www.hapmap.org). This effort has helped identify SNPs that are correlated extensively at the population level, i.e., SNPs that are in linkage disequilibrium (LD). Dueto the extensive correlations, it is not necessary to genotype all SNPs across a particular genomic region for an association study. Instead, representative SNPs, or 'tag SNPs', can be selected and genotyped. We used this approach. Common tag SNPs in an extended set of circadian genes were assayed in a large, national sample of BPI cases. A sample of SZ/SZA cases was also included. The SZ/SZA samples were intended not only to identify associations with these disorders, but also to provide comparisons with the BPI associations. Several studies have recently shown overlapping association between BPI and SZ/SZA, which may indicate shared genetic etiology (41-44). 


\section{Methods}

\section{Clinical}

BPI cases-Genomic DNA samples were obtained from BPI patients recruited through the Systematic Treatment Enhancement Program for Bipolar Disorder (STEP-BD) (45). STEPBD used a network of 18 US treatment centers for standardized evaluation and treatment of patients, including interview schedules based on the Structured Clinical Interview for DSMIV (SCID) (46), as well as the Mini-International Neuropsychiatric Interview (MINI) (47).

The selected samples represent a subset of the entire STEP-BD dataset. They were pruned by the following factors: consent for genetic studies, diagnosis of BPI, reported Caucasian ancestry, and samples released by the National Institute of Mental Health Genetics Research Initiative Repository (NIMH-GRI) at the time of the study.

SZ/SZA cases-Unrelated patients with SZ or SZA (according to DSM-IV criteria) were recruited at Western Psychiatric Institute and Clinic, Pittsburgh, PA, USA, and surrounding regions $(\mathrm{N}=527 ; \mathrm{n}=314 \mathrm{SZ}$ and $\mathrm{n}=213 \mathrm{SZA})$. Details of ascertainment and diagnosis are described elsewhere $(48,49)$. The Diagnostic Interview for Genetic Studies (DIGS), a structured diagnostic interview schedule (50), was used to interview the patients. Additional clinical information was obtained from clinical records and from relatives, as required. Consensus diagnoses were assigned following synthesis of the detailed data.

Control samples-Two sets of adult controls screened for absence of psychosis or BPI were included: a Pittsburgh-based sample and a national sample available from the NIMHGRI. Only control subjects reporting European-American ancestry were selected, in order to keep the control samples consistent with the case samples and to reduce spurious associations due to population substructure. The controls thus reflected the demographic distribution of the BPI patients, who were recruited at sites across the US, with a substantial proportion being recruited at Pittsburgh. The Pittsburgh-based controls were also suitable for comparison with the SZ/SZA cases. We wished to balance the control sample size to the case samples. The NIMH samples were released by the NIMH-GRI following a request for genomic DNA from individuals reporting Caucasian ancestry.

Pittsburgh controls ( $\mathbf{n}=\mathbf{1 6 8}$ ): Members of the local community were recruited through the University Center for Social and Urban Research (UCSUR), notices at a University of Pittsburgh Web site, or through advertisements in the local media. UCSUR is an independent center at the University of Pittsburgh experienced in performing telephone recruitment and screening services. UCSUR staff employed random digit-dialing sampling to ascertain potential control individuals. None of the subjects reported history of substance use disorder within the past month, serious medical or neurological illnesses, or mental retardation as defined in the DSM-IV. In addition to the above inclusion/exclusion criteria, individuals who reported a first-degree relative with psychoses or bipolar disorder were excluded.

NIMH-GRI controls $(\mathbf{n}=\mathbf{3 0 9})$ : Adults were accessed through a representative database maintained by a commercial firm. Consenting participants were assessed using a Web-based instrument regarding medical history, ancestral background, and previous diagnosis/ treatment for SZ, SZA, bipolar disorder, auditory hallucinations, or delusions (51-53). Further details are available on the study Web site (http://www.ncbi.nlm.nih.gov/projects/gap/cgi-bin/study.cgi?study_id=phs000021.v2.p1).

All participants reported Caucasian ethnicity. They provided written informed consent, according to the guidelines of the University of Pittsburgh Institutional Review Board. 


\section{Laboratory}

Gene selection-The PubMed database was searched using the key words 'circadian, clock, genes'. The search yielded 21 genes in December 2006 (Table 1).

SNP selection-We selected 276 'tag SNPs'; i.e., SNPs that would represent common variants across the selected genes. The regions represented included 5 kilobases $(\mathrm{kb})$ upstream and $5 \mathrm{~kb}$ downstream of the coding sequence, from the International HapMap Project Web site (http://www.hapmap.org/). All tag SNPs had minor allele frequencies (MAF) greater than 5\% in Caucasians, with correlation between SNPs below a predetermined threshold $\left(r^{2}<0.8\right)$.

Genotype assays-Genomic DNA was extracted from venous blood samples using the phenol chloroform method or using Puregene DNA extraction kits (http://www1.qiagen.com/Products/GenomicDnaStabilizationPurification/ GentraPuregeneBloodKit.aspx). Of the identified tag SNPs $(n=276), 268$ could be genotyped successfully using the multiplexed, hybridization-based Illumina Golden-Gate Assay (http://www.illumina.com) (54).

Quality control for genotype assays: Seventeen individuals from the Centre d'Etude du Polymorphism Humain (CEPH) genotyped in the HapMap project were used as 'positive controls', as their genotypes for particular SNPs is known from the HapMap database. In addition, 34 other samples (two/assay plate) were used by Illumina, Inc., as internal controls. Three SNPs from the X chromosome were used for gender checks (rs2040962, rs1108444, and rs988457). Out of 276 SNPs genotyped, 268 SNPS were evaluated further for association (excluding the X chromosome SNPs). Eight SNPs were dropped from analysis: three SNPs because of discordant genotypes with CEPH samples (having two or more discordant), four SNPs failing $100 \%$ of genotypes, and rs 895683 at ARNTL gene having a genotype failure rate over $2.5 \%$. For the remaining 268 SNPs, concordance between our genotype calls and HapMap calls was $100 \%$. The mean genotype call rate was $99.96 \%$. Deviations from Hardy-Weinberg Equilibrium (HWE) were evaluated for each SNP using a global significance threshold of $\mathrm{p}>0.005$.

\section{Statistical analysis}

LD was analyzed using Hclust software to select tag SNPs (55). Gene-based tests were conducted initially to test for associations, followed by tests with individual SNPs. For genebased analyses, we used the (T-max) test of association fitting additive, dominant, and recessive models for each gene (56). SNP-based association analyses for the diagnostic phenotype were conducted using the Cochran-Armitage trend test (57).

Tests of population substructure-We used a variation of the genomic control (GC) method (58-60). Samples in the present report were also genotyped for other studies. In sum, 711 SNPs were genotyped from 64 different genes, i.e., 64 generally independent genomic regions. We performed 10,000 iterations of randomly drawing a single SNP from each of the 64 genes to compare between cases and controls, obtaining a distribution of median chi-square tests. The mean value of this distribution was calculated and was divided by the expected median of a chi-square distribution with one degree of freedom $(0.456)$. Since control samples were obtained from two different geographic regions, we conducted identical analyses between the two control groups to assess within-group substructure. Based on our genomic control analysis (10,000 iterations comparing SNPs from 64 different genomic regions we analyzed), we found no differences between BPI cases and controls ( $\lambda$ $=0.85$ ). If population substructure leads to 'spurious' case-control differences, an inflation in the chi square value would be expected for the SNPs used for the genomic control 
analyses. We found a relatively small inflation in the test statistics between SZ/SZA cases and controls $(\lambda=1.08)$. Since our controls were obtained from two different sources, we also performed similar genomic control analyses between controls from Pittsburgh and the NIMH-GRI controls, and found the inflation factor to be smaller than for the SZ/SZA case/ control comparisons $(\lambda=1.04)$.

Power analysis-We evaluated the power of our sample to detect an odds ratio (OR) of 1.5 under a dominant model. We tested the assumptions that the risk allele was actually analyzed in our sample or that a surrogate was genotyped at $r^{2}$ of 0.8 with the risk loci (similar to our primary analyses). We assessed power using risk allele frequencies of 0.15 and 0.5 in the population, and set the type I error threshold to 0.05 .

\section{Results}

\section{Demographic details and overlap}

The sample included 523 patients with BPI $(\mathrm{n}=225$ men, 298 women; mean age $\pm \mathrm{SD}=$ $43.11 \pm 12.71)$, 527 with SZ/SZA ( $=340$ men, 187 women; age $=37.31 \pm 10.12$ ), and 477 control individuals $(n=229$ men, 248 women; age $=46.44 \pm 16.72)$. The BPI cases but not the controls reported here are also part of a published GWAS study (see details below) (15). The SZ/SZA sample included 291 patients reported in earlier analysis (40), but the BPI and control samples are independent of that sample.

\section{Gene-based tests}

Nominally significant associations were noted at EGR3 with the BPI sample under two models. The best fits were a recessive model $\left(\chi^{2}=7.248, \mathrm{p}=0.014\right)$ and additive model $\left(\chi^{2}\right.$ $=7.659, \mathrm{p}=0.02)$. Nominally significant associations were also noted with the SZ/SZA sample at $N P A S 2$ under a recessive model $\left(\chi^{2}=11.321, \mathrm{p}=0.034\right)$. Using a dominant model, a trend for association was noted at $R O R B$ with the SZ/SZA sample $\left(\chi^{2}=9.945, \mathrm{p}=\right.$ 0.056). All these associations are uncorrected for multiple comparisons. (See Supplementary Data Tables S1 and S2).

\section{Single SNP-based analyses}

Bipolar I disorder-Nominally significant associations ( $\mathrm{p}=0.05$ or better) were detected at 10 SNPs in six genes when genotype distributions were contrasted at individual SNPs between BPI cases and control individuals using the Trend's test [ARNTL SNPs rs7126303 ( $\mathrm{p}=0.040) ;$ Cry2: rs1554338 (p = 0.031); CSNK1E: rs1997644 (p=0.024); EGR3: rs1996147 ( $\mathrm{p}=0.006) ;$ NPAS2: rs17025005 ( $\mathrm{p}=0.009), \mathrm{rs} 13025524(\mathrm{p}=0.016)$, rs11123857 ( $\mathrm{p}=0.036)$; and RORB: $\mathrm{rs} 17691363(\mathrm{p}=0.035), \operatorname{rs} 10217594(\mathrm{p}=0.026)$, rs10491929 ( $=0.023)$; see Table 2].

Schizophrenia/schizoaffective disorder-Nominally significant associations were detected at eight SNPs across four genes: NPAS2 SNPs rs13025524 ( $\mathrm{p}=0.025), \mathrm{rs} 11123857$ $(\mathrm{p}=0.038), \operatorname{rs} 6543004(\mathrm{p}=0.024) ;$ PER2 SNP rs2304674 $(\mathrm{p}=0.033), \mathrm{rs} 2306472(\mathrm{p}=$ 0.048); PER3 SNP rs10462021 ( $\mathrm{p}=0.036)$, rs2640909 ( $\mathrm{p}=0.031)$; and RORB SNP rs10491929 ( $\mathrm{p}=0.023)$.

SNPs rs13025524 and rs11123857 (NPAS2); and rs10491929 (RORB) were nominally associated with both BPI and SZ/SZA ( $\mathrm{p}<0.05)$. All SNPs with nominally significant associations were localized to introns, except rs2304672 (PER2) and rs10462021 and rs2640909 (PER3) (all these SNPs are localized to exons; see Table 2). 


\section{Exploratory analyses}

Among the BPI cases, associations between SNPs and body mass index, age at onset, gender, and presence or absence of psychosis were tested. Nominally significant associations were detected across 14 genes (See Supplementary Data Table S3).

None of the above associations remained significant following Bonferoni corrections for multiple comparisons.

\section{Analyses in relation to GWAS}

The results from the STEP-BD (BPI) cases and controls were analyzed in relation to two independent GWAS reports that have been published recently $(13,15)$. The present samples overlapped with the Sklar et al. (15) study, so the present associations were evaluated primarily in relation to the Wellcome Trust Case Control Consortium (WTCCC) study (13). Both GWAS reported on identical Affymetrix GeneChip based assays but reported on slightly different sets of SNPs. Across the genomic regions analyzed here, the Sklar et al. study reported on 173 SNPs and the WTCCC analyzed 217 SNPs. Of the 268 SNPs analyzed here, 40 were reported in the Sklar et al. study (14.9\%), and 42 SNPs were analyzed in the WTCCC (15.6\%). SNP rs8031897 at FLJ20516 showed nominal evidence for association with BPI in our study $(\mathrm{p}=0.05)$ and a trend for association in the WTCCC study $(\mathrm{p}=0.07)$. SNP rs7126303 $(A R N T L)$ showed nominally significant association in our study $(\mathrm{p}=0.040)$. This SNP is in LD with two other SNPs that were associated with BPI at nominal levels in the WTCCC study $\left[\operatorname{rs} 3816360\left(r^{2}=0.73, \mathrm{p}=0.047\right)\right.$, rs7947951 $\left(r^{2}=\right.$ $0.536, \mathrm{p}=0.036)]$. Similar associations were not observed in the Sklar et al. (15) study. However, SNP rs 1554338 at $C R Y 2$ showed suggestive association with BPI in both our study $(\mathrm{p}=0.026)$ and the Sklar et al. $(15)$ study $(\mathrm{p}=0.0124)$.

\section{Power analysis}

Under a dominant model, the sample had $84.6 \%$ power to detect a nominally significant association (OR 1.5) for a risk allele with 15\% MAF in the population, using a type I error threshold of 5\%. For a risk allele with MAF 50\%, power declined to $74.7 \%$ under the same assumptions.

\section{Discussion}

We have systematically analyzed tag SNPs to enable a more comprehensive evaluation of circadian genes than has been reported. To our knowledge, this is the most extensive analysis of circadian gene polymorphisms to date, enabling us to evaluate a number of prior published associations simultaneously. We analyzed BPI cases, as well as SZ/SZA cases, using the same set of controls to evaluate associations with these disorders simultaneously. For associations with the diagnostic groups, we focused on gene-based tests. Such tests take account of correlations between SNPs due to LD and thus enable tests of overall associations in discrete genomic regions. Some novel, nominally significant associations were detected. For example, the gene-based tests indicated associations with BPI sample at $E G R 3$, a gene that is involved in the transcriptional regulation of other genes mediating biological rhythms (61). Suggestive associations were also detected with SZ/SZA sample at $N P A S 2$ and $R O R B$ genes. Several associations at individual SNPs were also detected at $N P A S 2$, which is localized to the frontal cortex, a region that may have a critical role in human circadian function (62). Prior reports have also suggested associations at NPAS2, but the patterns of associations noted in the present sample are different, so additional investigations are necessary. Despite the suggestive findings, since no association was significant after correcting for multiple testing, our gene-based and SNP based analyses indicate that individual common polymorphisms of the genes investigated are unlikely to 
confer substantial risk for BPI or SZ/SZA, i.e., ORs greater than 1.5. Replicate analyses using the two independent GWAS BPI samples did show direct replication of the specific SNPs that were suggestively associated with BPI, but the comparisons were limited by the modest overlap of the SNPs analyzed in both studies, although ARNTL and CRY2 genes each had SNPs with suggestive associations $(\mathrm{p}<0.05)$ in both the current study and at least one of the GWAS studies. Since our samples overlapped with the GWAS study by Sklar et al. (15), we evaluated our results primarily in relation to the WTCCC study (13).

The circadian gene hypothesis could be investigated through additional analyses. First, the relatively small effect sizes noted here indicate that it may be necessary to evaluate larger samples. Rare polymorphisms or copy number variations were not analyzed. We also did not evaluate haplotypes or epistatic interactions, in order to limit the number of comparisons. Risks due to haplotypes at BHLHB2, CSNK1E, and CLOCK genes have been suggested recently (34). We could not test this association, because only one SNP out of the three composing the relevant haplotype was genotyped in our study. It is also possible that other genes not analyzed in the present study also impact circadian function and are associated with BPI. Though a limited number of quantitative traits related to BPI were analyzed in the present study, it remains possible that there are undetected associations with other circadian traits that may underlie BPI pathogenesis. For example, we and others have replicably found that BPI patients are likely to differ from controls with regard to morningness/eveningness, a measure of preference for a stable, quantifiable measure that reflects preferred circadian phase (64).

In conclusion, we report on systematic analyses of selected circadian gene polymorphisms with BPI and SZ/SZA. Our data do not suggest substantial associations (OR > 1.5) with common polymorphisms of the genes analyzed here. Further studies using related circadian variables may be informative.

\section{Supplementary Material}

Refer to Web version on PubMed Central for supplementary material.

\section{Acknowledgments}

We thank the research participants for their generous help. This research was supported in part by grants from the National Institutes of Health (MH63420, MH56242 to VLN, Mental Health Intervention Research Center, MH30915 to DJK and MH45156). Recruitment of some of the Pittsburgh controls was funded through NIMH grants (MH56242, R01 MH060952-06). This publication was also supported by funds received from the c UL1 RR024153 and NIH/NCRR/GCRC Grant M01 RR00056 and Calabrese NIMH P20 MH-66054. We thank Drs. Raymond Cho, Gordon Frankle, Gretchen Haas, and the clinical core staff of the Center for the Neuroscience of Mental Disorders (MH45156) for their assistance in diagnostic and psychopathological assessments of some of the controls analyzed here.

\section{References}

1. Rao DC, Morton NE, Gottesman II, Lew R. Path analysis of qualitative data on pairs of relatives: application to schizophrenia. Hum Hered. 1981; 31:325-333. [PubMed: 7333621]

2. McGue M, Gottesman II, Rao DC. The transmission of schizophrenia under a multifactorial threshold model. Am J Hum Genet. 1983; 35:1161-1178. [PubMed: 6650500]

3. Tsuang, MT.; Faraone, SV. The genetic epidemiology of bipolar disorder. In: Marneros, A.; Angst, J., editors. Bipolar Disorders: 100 years After Manic-Depressive Insanity. Zurich: Kluwer Academic; 2000. p. 231-242.

4. Craddock N, Khodel V, Van Eerdewegh P, Reich T. Mathematical limits of multilocus models: the genetic transmission of bipolar disorder. Am J Hum Genet. 1995; 57:690-702. [PubMed: 7668299] 
5. Badner JA, Gershon ES. Meta-analysis of whole-genome linkage scans of bipolar disorder and schizophrenia. Mol Psychiatry. 2002; 7:405-411. [PubMed: 11986984]

6. Segurado R, Detera-Wadleigh SD, Levinson DF, et al. Genome scan meta-analysis of schizophrenia and bipolar disorder, part III: Bipolar disorder. Am J Hum Genet. 2003; 73:49-62. [PubMed: 12802785]

7. Risch N, Merikangas K. The future of genetic studies of complex human diseases. Science. 1996; 273:1516-1517. [PubMed: 8801636]

8. Berrettini W. Review of bipolar molecular linkage and association studies. Curr Psychiatry Rep. 2002; 4:124-129. [PubMed: 11914173]

9. Altshuler D, Daly MJ, Lander ES. Genetic mapping in human disease. Science. 2008; 322:881-888. [PubMed: 18988837]

10. Sullivan PF, Lin D, Tzeng JY, et al. Genomewide association for schizophrenia in the CATIE study: results of stage 1. Mol Psychiatry. 2008; 13:570-584. [PubMed: 18347602]

11. Pearson JV, Huentelman MJ, Halperin RF, et al. Identification of the genetic basis for complex disorders by use of pooling-based genomewide single-nucleotide-polymorphism association studies. Am J Hum Genet. 2007; 80:126-139. [PubMed: 17160900]

12. Lencz T, Lambert C, DeRosse P, et al. Runs of homozygosity reveal highly penetrant recessive loci in schizophrenia. Proc Natl Acad Sci USA. 2007; 104:19942-19947. [PubMed: 18077426]

13. The Wellcome Trust Case Control Consortium. Genome-wide association study of 14,000 cases of seven common diseases and 3,000 shared controls. Nature. 2007; 447:661-678. [PubMed: 17554300]

14. Baum AE, Akula N, Cabanero M, et al. A genome-wide association study implicates diacylglycerol kinase eta (DGKH) and several other genes in the etiology of bipolar disorder. Mol Psychiatry. 2008; 13:197-207. [PubMed: 17486107]

15. Sklar P, Smoller JW, Fan J, et al. Whole-genome association study of bipolar disorder. Mol Psychiatry. 2008; 13:558-569. [PubMed: 18317468]

16. Lenox RH, Gould TD, Manji HK. Endophenotypes in bipolar disorder. Am J Med Genet. 2002; 114:391-406. [PubMed: 11992561]

17. Mansour HA, Monk TH, Nimgaonkar VL. Circadian genes and bipolar disorder. Ann Med. 2005; 37:196-205. [PubMed: 16019718]

18. Kennedy SH, Kutcher SP, Ralevski E, Brown GM. Nocturnal melatonin and 24-hour 6sulphatoxymelatonin levels in various phases of bipolar affective disorder. Psychiatry Res. 1996; 63:219-222. [PubMed: 8878318]

19. Jones SH, Hare DJ, Evershed K. Actigraphic assessment of circadian activity and sleep patterns in bipolar disorder. Bipolar Disord. 2005; 7:176-186. [PubMed: 15762859]

20. Frank E, Swartz HA, Kupfer DJ. Interpersonal and social rhythm therapy: managing the chaos of bipolar disorder. Biol Psychiatry. 2000; 48:593-604. [PubMed: 11018230]

21. Salvatore $\mathrm{P}$, Ghidini S, Zita G, et al. Circadian activity rhythm abnormalities in ill and recovered bipolar I disorder patients. Bipolar Disord. 2008; 10:256-265. [PubMed: 18271904]

22. Roybal K, Theobold D, Graham A, et al. Mania-like behavior induced by disruption of CLOCK. Proc Natl Acad Sci USA. 2007; 104:6406-6411. [PubMed: 17379666]

23. Klein PS, Melton DA. A molecular mechanism for the effect of lithium on development. Proc Natl Acad Sci USA. 1996; 93:8455-8459. [PubMed: 8710892]

24. Bosch F, Gomez-Foix AM, Arino J, Guinovart JJ. Effects of lithium ions on glycogen synthase and phosphorylase in rat hepatocytes. J Biol Chem. 1986; 261:16927-16931. [PubMed: 3023372]

25. Klemfuss H. Rhythms and the pharmacology of lithium. Pharmacol Ther. 1992; 56:53-78. [PubMed: 1297145]

26. Klemfuss H, Kripke DF. Antimanic drugs stabilize hamster circadian rhythms. Psychiatry Res. 1995; 57:215-222. [PubMed: 7501731]

27. Benedetti F, Serretti A, Colombo C, Lorenzi C, Tubazio V, Smeraldi E. A glycogen synthase kinase 3-beta promoter gene single nucleotide polymorphism is associated with age at onset and response to total sleep deprivation in bipolar depression. Neurosci Lett. 2004; 368:123-126. [PubMed: 15351432] 
28. Szczepankiewicz A, Skibinska M, Hauser J, et al. Association analysis of the GSK-3beta T-50C gene polymorphism with schizophrenia and bipolar disorder. Neuropsychobiology. 2006; 53:5156. [PubMed: 16397405]

29. Lee KY, Ahn YM, Joo EJ, et al. No association of two common SNPs at position -1727 A/T, -50 C/T of GSK-3 beta polymorphisms with schizophrenia and bipolar disorder of Korean population. Neurosci Lett. 2006; 395:175-178. [PubMed: 16289845]

30. Nishiguchi N, Breen G, Russ C, St Clair D, Collier D. Association analysis of the glycogen synthase kinase-3beta gene in bipolar disorder. Neurosci Lett. 2006; 394:243-245. [PubMed: 16289783]

31. Nievergelt CM, Kripke DF, Barrett TB, et al. Suggestive evidence for association of the circadian genes PERIOD3 and ARNTL with bipolar disorder. Am J Med Genet B Neuropsychiatr Genet. 2006; 141B:234-241. [PubMed: 16528748]

32. Nievergelt CM, Kripke DF, Remick RA, et al. Examination of the clock gene Cryptochrome 1 in bipolar disorder: mutational analysis and absence of evidence for linkage or association. Psychiatr Genet. 2005; 15:45-52. [PubMed: 15722957]

33. Shiino Y, Nakajima S, Ozeki Y, Isono T, Yamada N. Mutation screening of the human period 2 gene in bipolar disorder. Neurosci Lett. 2003; 338:82-84. [PubMed: 12565145]

34. Shi J, Wittke-Thompson JK, Badner JA, et al. Clock genes may influence bipolar disorder susceptibility and dysfunctional circadian rhythm. Am J Med Genet B Neuropsychiatr Genet. 2008; 147B:1047-1055. [PubMed: 18228528]

35. Martinek S, Inonog S, Manoukian AS, Young MW. A role for the segment polarity gene shaggy/ GSK-3 in the Drosophila circadian clock. Cell. 2001; 105:769-779. [PubMed: 11440719]

36. Hansen L, Arden KC, Rasmussen SB, et al. Chromosomal mapping and mutational analysis of the coding region of the glycogen synthase kinase-3alpha and beta isoforms in patients with NIDDM. Diabetologia. 1997; 40:940-946. [PubMed: 9267989]

37. Kishi T, Kitajima T, Ikeda M, et al. Association analysis of nuclear receptor Reverb alpha gene (NR1D1) with mood disorders in the Japanese population. Neurosci Res. 2008; 62:211-215. [PubMed: 18804497]

38. Martin JL, Jeste DV, Ancoli-Israel S. Older schizophrenia patients have more disrupted sleep and circadian rhythms than age-matched comparison subjects. J Psychiatr Res. 2005; 39:251-259. [PubMed: 15725423]

39. Wirz-Justice A, Haug HJ, Cajochen C. Disturbed circadian rest-activity cycles in schizophrenia patients: an effect of drugs? Schizophr Bull. 2001; 27:497-502. [PubMed: 11596850]

40. Mansour H, Wood J, Logue T, et al. Association study of eight circadian genes with bipolar I disorder, schizoaffective disorder and schizophrenia. Genes Brain Behav. 2006; 5:150-157. [PubMed: 16507006]

41. Owen MJ, Craddock N, Jablensky A. The genetic deconstruction of psychosis. Schizophr Bull. 2007; 33:905-911. [PubMed: 17551090]

42. Perlis RH, Purcell S, Fagerness J, et al. Family-based association study of lithium-related and other candidate genes in bipolar disorder. Arch Gen Psychiatry. 2008; 65:53-61. [PubMed: 18180429]

43. Goghari VM, Sponheim SR. Differential association of the COMT Val158Met polymorphism with clinical phenotypes in schizophrenia and bipolar disorder. Schizophr Res. 2008; 103:186-191. [PubMed: 18571901]

44. Serretti A, Mandelli L. The genetics of bipolar disorder: genome 'hot regions,' genes, new potential candidates and future directions. Mol Psychiatry. 2008; 13:742-771. [PubMed: 18332878]

45. Sachs GS, Thase ME, Otto MW, et al. Rationale, design, and methods of the systematic treatment enhancement program for bipolar disorder (STEP-BD). Biol Psychiatry. 2003; 53:1028-1042. [PubMed: 12788248]

46. Spitzer, R.; Williams, J.; Gibbon, M. Structured Clinical Interview for DSM-IV, Outpatient Version (SCID-OP). New York: Biometrics Research Department, New York State Psychiatric Institute; 1996. 
47. Sheehan DV, Lecrubier Y, Sheehan KH, et al. The Mini-International Neuropsychiatric Interview (M.I.N.I): the development and validation of a structured diagnostic psychiatric interview for DSM-IV and ICD-10. J Clin Psychiatry. 1998; 59(Suppl 20):22-33. [PubMed: 9881538]

48. Chowdari KV, Mirnics K, Semwal P, et al. Association and linkage analyses of RGS4 polymorphisms in schizophrenia. Hum Mol Genet. 2002; 11:1373-1380. [PubMed: 12023979]

49. Talkowski ME, Mansour H, Chowdari KV, et al. Novel, replicated associations between dopamine D3 receptor gene polymorphisms and schizophrenia in two independent samples. Biol Psychiatry. 2006; 60:570-577. [PubMed: 16893532]

50. Nurnberger JI Jr, Blehar MC, Kaufmann CA, et al. Diagnostic interview for genetic studies. Rationale, unique features, and training. NIMH Genetics Initiative. Arch Gen Psychiatry. 1994; 51:849-859. [PubMed: 7944874]

51. Sanders AR, Duan J, Levinson DF, et al. No significant association of 14 candidate genes with schizophrenia in a large European ancestry sample: implications for psychiatric genetics. Am J Psychiatry. 2008; 165:497-506. [PubMed: 18198266]

52. Suarez BK, Duan J, Sanders AR, et al. Genomewide linkage scan of 409 European-ancestry and African American families with schizophrenia: suggestive evidence of linkage at 8p23.3-p21.2 and 11p13.1-q14.1 in the combined sample. Am J Hum Genet. 2006; 78:315-333. [PubMed: $16400611]$

53. Manolio TA, Rodriguez LL, Brooks L, et al. New models of collaboration in genome-wide association studies: the Genetic Association Information Network. Nat Genet. 2007; 39:10451051. [PubMed: 17728769]

54. Shen R, Fan JB, Campbell D, et al. High-throughput SNP genotyping on universal bead arrays. Mutat Res. 2005; 573:70-82. [PubMed: 15829238]

55. Rinaldo A, Bacanu SA, Devlin B, Sonpar V, Wasserman L, Roeder K. Characterization of multilocus linkage disequilibrium. Genet Epidemiol. 2005; 28:193-206. [PubMed: 15637716]

56. Roeder K, Bacanu SA, Sonpar V, Zhang X, Devlin B. Analysis of single-locus tests to detect gene/ disease associations. Genet Epidemiol. 2005; 28:207-219. [PubMed: 15637715]

57. Armitage P. Tests for linear trends in proportions and frequencies. Biometrics. 1955; 11:375-386.

58. Devlin B, Roeder K. Genomic control for association studies. Biometrics. 1999; 55:997-1004. [PubMed: 11315092]

59. Bacanu SA, Devlin B, Roeder K. The power of genomic control. Am J Hum Genet. 2000; 66:1933-1944. [PubMed: 10801388]

60. Devlin B, Bacanu SA, Roeder K. Genomic control to the extreme. Nat Genet. 2004; 36:11291130. [PubMed: 15514657]

61. Morris ME, Viswanathan N, Kuhlman S, Davis FC, Weitz CJ. A screen for genes induced in the suprachiasmatic nucleus by light. Science. 1998; 279:1544-1547. [PubMed: 9488654]

62. Reick M, Garcia JA, Dudley C, McKnight SL. NPAS2: an analog of clock operative in the mammalian forebrain. Science. 2001; 293:506-509. [PubMed: 11441147]

63. Johansson C, Willeit M, Smedh C, et al. Circadian clock-related polymorphisms in seasonal affective disorder and their relevance to diurnal preference. Neuropsychopharmacology. 2003; 28:734-739. [PubMed: 12655319]

64. Wood J, Birmaher B, Axelson D, et al. Replicable differences in preferred circadian phase between bipolar disorder patients and control individuals. Psychiatry Res. 2009; 166:201-209. [PubMed: 19278733] 


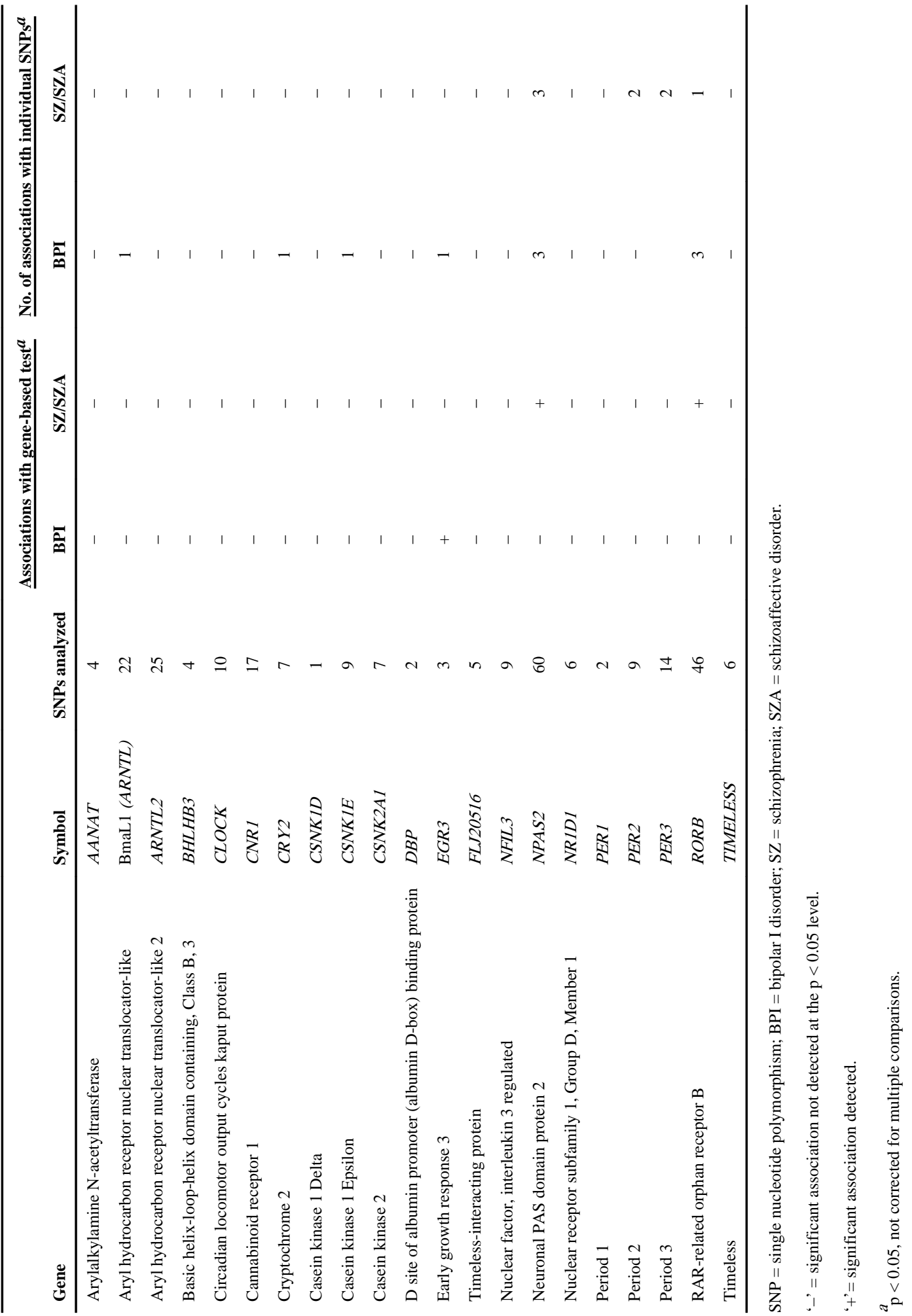

Bipolar Disord. Author manuscript; available in PMC 2012 July 23. 
